K. Mimachi

Nagoya Math. J.

Vol. 116 (1989), 149-161

\title{
CONNECTION PROBLEM IN HOLONOMIC $q$-DIFFERENCE SYSTEM ASSOCIATED WITH A JACKSON INTEGRAL OF JORDAN-POCHHAMMER TYPE
}

\author{
KATSUHISA MIMACHI
}

\section{§. Introduction}

Fix a complex number $q$ with $|q|<1$. Let $T_{1}, \cdots, T_{n}$ be $n$-commuting $q$-difference operators defined by

$$
T_{j} f\left(x_{1}, \cdots, x_{n}\right)=f\left(x_{1}, \cdots, q x_{j}, \cdots, x_{n}\right)
$$

for a function $f(x), x=\left(x_{1}, \cdots, x_{n}\right) \in\left(C^{*}\right)^{n}$. Consider a system of linear $q$-difference equations in several variables for a matrix valued function $\Xi(x)$ on $\left(C^{*}\right)^{n}$ as follows:

$$
T_{i} \Xi(x)=\Xi(x) A_{i}(x) \quad(1 \leq i \leq n) .
$$

We assume that each $A_{i}(x)$ is a matrix valued rational function satisfying the following conditions:

$$
A_{i}(x) T_{i} A_{j}(x)=A_{j}(x) T_{j} A_{i}(x) \quad(1 \leq i, j \leq n) .
$$

Then (1) defines a holonomic q-difference system. It is known [3] that there is a solution of the system (1) characterized by asymptotic behavior at a boundary point of $\left(C^{*}\right)^{n}$. More precisely, we denote by $L_{\nu}(x)=$ $\left\{\left(q^{\nu_{1} m} x_{1}, \cdots, q^{\nu_{n} m} x_{n}\right) \mid m \in Z\right\}$ the trajectory through $x \in\left(C^{*}\right)^{n}$ of the transformation $\left(y_{1}, \cdots, y_{n}\right) \rightarrow\left(q^{\nu_{1}} y_{1}, \cdots, q^{\nu_{n}} y_{n}\right)$ which is determined by integers $\nu=\left(\nu_{1}, \cdots, \nu_{n}\right)$. Under Ass 1 and Ass 2 in the direction $L_{\nu}$ for $A_{i}(x)$ stated in [3] the above solution denoted by $E_{\nu}(x)$ is characterized by the following asymptotic behavior along $L_{\nu}$ at $m=\infty$ :

$$
\begin{aligned}
& E_{\nu}(x) \sim \bar{x}_{1}^{\Lambda_{1}} \bar{x}_{2}^{\Lambda_{2}} \cdots \bar{x}_{n}^{\Lambda_{n}} \cdot U_{\nu}, \\
& \quad \bar{x}_{1}=q^{\nu_{1} m} x_{1}, \cdots, \bar{x}_{n}=q^{\nu_{n} m} x_{n}, \quad \text { as } m \rightarrow \infty,
\end{aligned}
$$

where $U_{\nu}$ denotes a certain non-singular lower triangular matrix which

Received August 23, 1988. 
is constant, and $\Lambda_{1}, \cdots, \Lambda_{n}$ denote constant diagonal matrices. We call $\Xi_{\nu}(x)$ the solution determined along the trajectory $L_{\nu}$. We take two trajectories $L_{\nu}$ and $L_{\mu}$ for two sequences of integers $\nu$ and $\mu$. Then there exists a linear relation between the corresponding solutions $\Xi_{\nu}(x)$ and $\Xi_{\mu}(x)$ along the trajectories $L_{\nu}$ and $L_{\mu}$ respectively as follows:

$$
\Xi_{\nu}(x)=P_{\nu, \mu}(x) \Xi_{\mu}(x),
$$

where $P_{\nu, \mu}(x)$ denotes a matrix valued function satisfying

$$
T_{j} P_{\nu, \mu}(x)=P_{\nu, \mu}(x) \quad(1 \leq j \leq n) .
$$

The matrix $P_{\nu, \mu}(x)$ is called a connection matrix between the two solutions determined by the trajectories $L_{\nu}$ and $L_{\mu}$.

The main purpose of this paper is to solve the connection problem, namely to compute the matrices $P_{\nu, \mu}(x)$, in holonomic $q$-difference system associated with a Jackson integral of Jordan-Pochhammer type under a generic condition. Jordan-Pochhammer type is a natural extension of Heine's basic hypergeometric series.

The contents of this paper are as follows. Section 1 gives basic notation in the $q$-analysis and a short review of a system associated with a Jackson integral of Jordan-Pochhammer type. Section 2 is devoted to give relations among Jackson integrals over suitable $q$-intervals of the first kind, which play a key role in our argument. We remark that, as a bonus of these relations, a connection formula of the basic hypergeometric series is obtained. In Section 3 we compute asymptotic behavior of the solutions along generic trajectories, and solve the corresponding connection problem.

\section{§1. Preliminaries}

Fix a complex number $q$ with $|q|<1$. Following F.H. Jackson [12], for a nonzero complex number $c \in C^{*}$, define on a half-line $[0, c]$

$$
\int_{[0, c]} F(t) d_{q} t=\int_{0}^{c} F(t) d_{q} t=c(1-q) \sum_{n \geq 0} F\left(c q^{n}\right) q^{n},
$$

which is a $q$-analogue of the Riemann integral and is called a Jackson integral. We also consider a Jackson integral on a whole line $[0, \infty(s)]$

$$
\int_{[0, \infty(s)]} F(t) d_{q} t=\int_{0}^{\infty(s)} F(t) d_{q} t=s(1-q) \sum_{-\infty \leq n \leq+\infty} F\left(s q^{n}\right) q^{n},
$$

for a complex number $s \in C^{*}$. We shall call $[0, c]$ a $q$-interval of the first 
kind or of the second kind according as $c \in C^{*}$ or $c=\infty(s)$. The following is easily deduced.

$$
\int_{0}^{c} F(t) d_{q} t=c \int_{0}^{1} F(c t) d_{q} t .
$$

Here we define a Jackson integral of Jordan-Pochhammer type by

$$
\int_{\mathscr{C}} t^{\alpha_{0}-1} \prod_{1 \leq j \leq n} \frac{\left(t / x_{j}\right)_{\infty}}{\left(q^{\alpha_{j}} t / x_{j}\right)_{\infty}} d_{q} t
$$

where $(a)_{\infty}=\prod_{n \geq 0}\left(1-a q^{n}\right), \alpha_{j} \in C(0 \leq j \leq n)$ and $\mathscr{C}$ denotes a suitable $q$-interval. The Jackson integral (3) tends to a Jordan-Pochhammer type integral:

$$
\int t^{\alpha_{0}-1} \prod_{1 \leq j \leq n}\left(1-\frac{t}{x_{j}}\right)^{\alpha_{j}} d t
$$

as $q \rightarrow 1$. In fact, if $(a)_{n}=(a)_{\infty} /\left(a q^{n}\right)_{\infty}$, then we have

$$
\lim _{q \rightarrow 1} \frac{(t)_{\infty}}{\left(q^{\alpha} t\right)_{\infty}}=(1-t)^{\alpha},
$$

by the $q$-binomial theorem ([2], [7])

$$
\sum_{n \geq 0} \frac{(a)_{n}}{(q)_{n}} x^{n}=\frac{(a x)_{\infty}}{(x)_{\infty}} .
$$

The holonomic q-difference system associated with a Jackson integral of Jordan-Pochhammer type is given as follows. Set

$$
\begin{aligned}
& \Phi(t)=t^{\alpha_{0}-1} \prod_{0 \leq j \leq n} \frac{\left(t / x_{j}\right)_{\infty}}{\left(q^{\alpha_{j}} t / x_{j}\right)_{\infty}}, \\
& \Phi_{j}(t)=\Phi(t) /\left(1-t / x_{j}\right) .
\end{aligned}
$$

The following lemma has been communicated to the author by Prof. $\mathrm{K}$. Aomoto ([4]):

LEMma 1. 1) A holonomic $q$-difference system for the function $\int \Phi d_{q} t$ can be derived in an explicit way

(5) $\quad T_{k}\left(\int \Phi_{1} d_{q} t, \cdots, \int \Phi_{n} d_{q} t\right)=\left(\int \Phi_{1} d_{q} t, \cdots, \int \Phi_{n} d_{q} t\right) A_{k} \quad(1 \leq k \leq n)$. 
Here each $A_{k}=\left(a_{i, j}^{(k)}\right)_{1 \leq i, j \leq n}$ is an $n \times n$ matrix valued function of $x$ with the entries which are rational in $x$

$$
\begin{aligned}
& a_{i, j}^{(k)}=q^{\alpha_{0}} \frac{\left(1-q^{\alpha_{k}}\right) \prod_{\substack{1 \leq l \leq n \\
l \neq k}}\left(1-\frac{x_{i}}{x_{l}} q^{\alpha_{l}}\right)}{\left(q \frac{x_{k}}{x_{j}}-q^{\alpha_{k}}\right) \prod_{\substack{1 \leq l \leq n \\
l \neq i}}\left(1-\frac{x_{i}}{x_{l}}\right)} \quad(i, j \neq k, i \neq j), \\
& a_{i, k}^{(k)}=q^{\alpha_{0}} \frac{\prod_{\substack{1 \leq l \leq n \\
l \neq k}}\left(1-\frac{x_{i}}{x_{l}} q^{\alpha_{i}}\right)}{\prod_{\substack{\leq l \leq n \\
l \neq i}}\left(1-\frac{x_{i}}{x_{l}}\right)} \quad(i \neq k), \\
& a_{i, i}^{(k)}=\frac{1-\frac{x_{i}}{q x_{k}}}{1-\frac{x_{i}}{x_{k}} q^{\alpha_{k}-1}}+q^{\alpha_{0}} \frac{\left(1-q^{\alpha_{i}}\right) \prod_{\substack{1 \leq l \leq n \\
l \neq k}}\left(1-\frac{x_{i}}{x_{l}} q^{\alpha_{l}}\right)}{\left(q \frac{x_{k}}{x_{i}}-q^{\alpha_{k}}\right) \prod_{\substack{1 \leq l \leq n \\
l \neq i}}\left(1-\frac{x_{i}}{x_{l}}\right)} \quad(i \neq k), \\
& a_{k, j}^{(k)}=q^{\alpha_{0}} \frac{\prod_{1 \leq l \leq n}\left(1-\frac{x_{k}}{x_{l}} q^{\alpha_{l}}\right)}{\left(q \frac{x_{k}}{x_{j}}-q^{\alpha_{k}}\right) \prod_{\substack{1 \leq l \leq n \\
l \neq k}}\left(1-\frac{x_{k}}{x_{l}}\right)} \quad(k \neq j), \\
& a_{k, k}^{(k)}=q^{\alpha_{0}} \prod_{\substack{1 \leq l \leq n \\
l \neq k}} \frac{1-\frac{x_{k}}{x_{l}} q^{\alpha_{l}}}{1-\frac{x_{k}}{x_{l}}} .
\end{aligned}
$$

2) A fundamental solution matrix is given by

$$
\Xi(x)=\left(\Xi_{i, j}\right)_{1 \leq i, j \leq n}=\left(\int_{w_{i}} \Phi_{j}(t) d_{q} t\right)_{1 \leq i, j \leq n},
$$

where $w_{i}$ denotes a q-interval $\left[0, x_{j}\right]$ for $1 \leq i \leq n$.

We investigate the behavior of $\Xi\left(q^{\nu_{1} m} x_{1}, \cdots, q^{\nu_{n} m} x_{n}\right)$ at $m=\infty$ along all generic trajectories $L_{\nu}$ determined by the $n$ ! inequalities $\nu_{\sigma(1)}<\nu_{\sigma(2)}<$ $\ldots<\nu_{\sigma(n)}$ where $\sigma$ run over the symmetric group of order $n$. If we put $\bar{x}_{1}=x_{1} q^{\nu_{1} m}, \cdots, \bar{x}_{n}=x_{n} q^{\nu_{n} m}$ for $x_{j} \in C^{*}$, then the condition $\nu_{\sigma(1)}<\cdots<\nu_{\sigma(n)}$ is equivalent to the condition $\left|\bar{x}_{\sigma(1)}\right| \gg \cdots \gg\left|\bar{x}_{\sigma(n)}\right|$ when $m$ is sufficiently large. Therefore the connection problem is reduced to find a relation between $\Xi(x)$ in the region $\left|x_{1}\right| \gg \cdots \gg\left|x_{n}\right| \gg 1$ and $\Xi(x)$ in the region $\left|x_{\sigma(1)}\right| \gg \cdots \gg\left|x_{\sigma(n)}\right| \gg 1$, which will be denoted by $\Xi_{e}(x)$ and $\Xi_{\sigma}(x)$ respectively. 


\section{§ 2. Relations among Jackson integrals of the first kind}

In this section we give relations among Jackson integrals of JordanPochhammer type, which will be essential in the sequel, and also give a connection formula for the basic hypergeometric series as its corollary. See [7], [15], [16] for related formulae.

We shall frequently use the theta function $\Theta(t)=(t)_{\infty}(q / t)_{\infty}(q)_{\infty}$.

Lemma 2. Let $k=1, \cdots, n-1$. Under the condition $\left|q^{-\alpha_{k}-\cdots-\alpha_{n}}\right|<$ $\left|q^{\alpha_{0}}\right|<1$ and $x_{i} \mid x_{j} \neq 1, q^{ \pm 1}, q^{ \pm 2}, \cdots$, we have relations

$$
\begin{gathered}
\int_{w_{k}} t^{\alpha_{0}-1} \prod_{j=k}^{n} \frac{\left(t / x_{j}\right)_{\infty}}{\left(q^{\alpha_{j}} t / x_{j}\right)_{\infty}} d_{q} t+\sum_{l=k+1}^{n} C_{k, l} \int_{w_{l}} t^{\alpha_{0}-1} \sum_{j=k}^{n} \frac{\left(t / x_{j}\right)_{\infty}}{\left(q^{\alpha_{j}} t / x_{j}\right)_{\infty}} d_{q} t \\
=x_{k}^{\alpha_{0}} \frac{\Theta\left(q^{1+\alpha_{0}+\alpha_{k}+\cdots+\alpha_{n}}\right)}{\Theta\left(q^{\alpha_{0}+\alpha_{k}+1+\cdots+\alpha_{n}}\right)} \prod_{j=k+1}^{n} \frac{\Theta\left(x_{j} / x_{k}\right)}{\Theta\left(x_{j} / x_{x} q^{\alpha_{j}}\right)} \\
\quad \times \int_{[0,1]} t^{-1-\alpha_{0}-\alpha_{k}-\cdots-\alpha_{n}} \prod_{j=k}^{n} \frac{\left(x_{j} x_{k}^{-1} q^{\alpha_{k}-\alpha_{j}} t\right)_{\infty}}{\left(x_{j} x_{k}^{-1} q^{\alpha_{k}} t\right)_{\infty}} d_{q} t,
\end{gathered}
$$

where

$$
C_{k, l}=\left(\frac{x_{k}}{x_{l}}\right)^{\alpha_{0}+1} \frac{\Theta\left(x_{k} \cdot x_{l}^{-1} q^{\alpha_{0}+\alpha_{k+1}+\cdots+\alpha_{n}+1}\right) \prod_{j=k+1}^{n} \Theta\left(x_{i} / x_{k}\right) \Theta\left(x_{l} q^{\alpha_{i}+1} / x_{i}\right)}{\Theta\left(q^{\alpha_{0}+\alpha_{k+1}+\cdots+\alpha_{n}+1}\right) \prod_{\substack{k \leq i \leq n \\ i \neq l}} \Theta\left(x_{i} / x_{l}\right) \prod_{i=k+1}^{n} \Theta\left(x_{k} q^{\alpha_{i}+1} / x_{i}\right)} .
$$

Remark. If a function $F(x)=\prod_{1 \leq i \leq n} x_{\imath}^{\lambda_{i}} f(x)$, where $f(x)$ is a meromorphic function on $\left(C^{*}\right)^{n}$, satisfies $T_{j} F(x)=F(x)$ for $j=1, \cdots, n$, then we say $F(x)$ to be pseudo-constant, which is also said to be $q$-periodic by C.R. Adams, G.D. Birkhoff, R.D. Carmichael, and W.J. Trjitzinsky ([1], [5], [6], [9]). The above functions $C_{k, l}(1 \leq k \leq n-1, k+1 \leq l \leq n)$ are pseudo-constant.

Proof of Lemma 2. We show these relations by residue calculus, which is an extension of the method as in our previous paper [13]. Set

$$
F(t)=\frac{\left(q^{-\alpha_{0}-\alpha_{k}-\cdots-\alpha_{n}-1} / t\right)_{\infty}\left(q^{\alpha_{0}+\alpha_{k}+\cdots+\alpha_{n}+2} t\right)_{\infty}}{(1 / t)_{\infty}\left(q^{\alpha_{k}+1} t\right)_{\infty}} \prod_{l=k+1}^{n} \frac{\left(x_{k}^{-1} x_{l} q^{\alpha_{k}+1-\alpha_{l}} t\right)_{\infty}}{\left(x_{k}^{-1} x_{l} q^{\alpha_{k}+1} t\right)_{\infty}},
$$

and

$$
\tilde{F}(t)=x_{k}^{\alpha_{0}} q^{\alpha_{0}+a_{k}+1} \frac{(1-q)(q)_{\infty}^{2}}{\Theta\left(q^{\alpha_{0}+a_{k+1}+\cdots+\alpha_{n}+1}\right)} \prod_{i=k+1}^{n} \frac{\Theta\left(x_{i} / x_{k}\right)}{\Theta\left(x_{i} / x_{k} q^{\alpha_{i}}\right)} F(t) .
$$

Then $F(t)$ is a meromorphic function on $C^{*}$. The residues of $\tilde{F}(t)$ at each point $q^{-1-\alpha_{k}-j}, x_{k} x_{l}^{-1} q^{-1-\alpha_{k}-j}, q^{i}(j=0,1,2, \cdots)$ are expressed by the following Jackson integrals. 


$$
\begin{aligned}
& \sum_{j \geq 0} \underset{t=q^{-1-\alpha_{k}-j}}{\operatorname{Res}} \tilde{F}(t)=-\int_{w_{k}} t^{\alpha_{0}-1} \prod_{i=k}^{n} \frac{\left(t / x_{i}\right)_{\infty}}{\left(q^{\alpha} t / x_{i}\right)_{\infty}} d_{q} t, \\
& \sum_{j \geq 0} \operatorname{Res}_{t=x_{k} x_{l}^{-1} q^{-1-\alpha_{k}-j}} \tilde{F}(t)=-C_{k, l} \int_{w_{l}} t^{\alpha_{0}-1} \prod_{j=k}^{n} \frac{\left(t / x_{j}\right)_{\infty}}{\left(q^{\alpha_{j}} t / x_{j}\right)_{\infty}} d_{q} t
\end{aligned}
$$

and

$$
\begin{aligned}
\sum_{j \geq 0} \operatorname{Res}_{t=q^{j}} \tilde{F}(t)= & x_{k}^{\alpha_{0}} \frac{\Theta\left(q^{1+\alpha_{0}+\alpha_{k}+\cdots+\alpha_{n}}\right)}{\Theta\left(q^{\alpha_{0}+\alpha_{k+1}+\cdots+\alpha_{n}}\right)} \prod_{j=k+1}^{n} \frac{\Theta\left(x_{j} / x_{k}\right)}{\Theta\left(x_{j} / x_{k} q^{\alpha_{j}}\right)} \\
& \times \int_{[0,1]} t^{-1-\alpha_{0}-\alpha_{k}-\cdots-\alpha_{n}} \sum_{j=k}^{n} \frac{\left(x_{j} x_{k}^{-1} q^{\alpha_{k}-\alpha_{j}} t\right)_{\infty}}{\left(x_{j} x_{k}^{-1} q^{\alpha_{k}} t\right)_{\infty}} d_{q} t,
\end{aligned}
$$

where $\operatorname{Res}_{t=x} F(t)$ denotes the residue of a function $F(t)$ at $t=x$. Therefore it remains to prove

$$
\sum_{j \geq 0} \underset{t=q^{-1-\alpha_{k}-j}}{\operatorname{Res}} F(t)+\sum_{l=k+1}^{n} \sum_{j \geq 0} \underset{t=x_{k} x_{l}^{-1} q^{-1-\alpha_{k}-j}}{\operatorname{Res}} F(t)+\sum_{j \geq 0} \operatorname{Res}_{t=q^{j}} F(t)=0 .
$$

Here we set two circles $\mathscr{C}_{m}, \tilde{\mathscr{C}}_{m}$ for a natural number $m$ as follows:

$$
\begin{aligned}
& \mathscr{C}_{m}:=\left\{\rho_{m} \exp (\varphi \sqrt{-1}) \mid \rho_{m}:=\frac{1}{2}\left(|q|^{m}+|q|^{m+1}\right), 0 \leq \varphi \leq 2 \pi\right\}, \\
& \tilde{\mathscr{C}}_{m}:=\left\{\tilde{\rho}_{m} \exp (\varphi \sqrt{-1})\left|\tilde{\rho}_{m}:=\frac{1}{2}\right| q^{-\alpha_{k}-1} \mid\left(|q|^{-m-1}+|q|^{-m}\right), 0 \leq \varphi \leq 2 \pi\right\},
\end{aligned}
$$

with the counterclockwise direction. Then we have

$$
\begin{gathered}
\sum_{j=0}^{m} \operatorname{Res}_{t=q^{-1-\alpha_{k}-j}} F(t)+\sum_{l=k+1}^{n} \sum_{j=0}^{m(l)} \underset{t=x_{k} x_{l}^{-1} q^{-1-\alpha_{k}-j}}{\operatorname{Res}} F(t)+\sum_{j=0}^{m} \operatorname{Res}_{t=q^{j}} F(t) \\
=\frac{1}{2 \pi \sqrt{-1}} \int_{\tilde{\mathscr{C}}_{m}} F(t) d t-\frac{1}{2 \pi \sqrt{-1}} \int_{\mathscr{C}_{m}} F(t) d t
\end{gathered}
$$

where each $m(l)(l=k+1, \cdots, n)$ is a certain positive integer. And theer exists a positive number $M$ such that

$$
\left|F\left(\rho_{m} e^{\sqrt{-1} \varphi}\right)\right| \leq M\left|q^{-1-\alpha_{0}-\alpha_{k}-\cdots-\alpha_{n}}\right|^{m}
$$

for $0 \leq \varphi \leq 2 \pi$. Indeed

$$
\begin{aligned}
& F\left(\rho_{m} e^{\sqrt{-1} \varphi}\right)=F\left(\rho_{0}|q|^{m} e^{\sqrt{-1} \varphi}\right) \\
& =\left(q^{-1-\alpha_{0}-\alpha_{k}-\cdots-\alpha_{n}}\right)^{m} \frac{\left(q^{2-m+\alpha_{0}+\alpha_{k}+\cdots+\alpha_{n}}|q|^{m} \rho_{0} e^{\sqrt{-1} \varphi}\right)_{m}}{\left(q^{1-m}|q|^{m} \rho_{0} e^{\sqrt{-1} \varphi}\right)_{m}} \\
& \quad \times \frac{\left(\left.q^{m-1-\alpha_{0}-\alpha_{k}-\cdots-\alpha_{n}}|| q\right|^{m} \rho_{0} e^{\sqrt{-1} \varphi}\right)_{\infty}\left(q^{2+\alpha_{0}+\alpha_{k}+\cdots+\alpha_{n}}|q|^{m} \rho_{0} e^{\sqrt{-1} \varphi}\right)_{\infty}}{\left(q^{m} /|q|^{m} \rho_{0} e^{\sqrt{-1} \varphi}\right)_{\infty}\left(q^{1+\alpha_{k}}|q|^{m} \rho_{0} e^{\sqrt{-1} \varphi}\right)^{\infty}}
\end{aligned}
$$




$$
\times \prod_{l=k+1}^{n} \frac{\left(x_{k}^{-1} x_{l} q^{1+\alpha_{k}-\alpha_{l}}|q|^{m} \rho_{0} e^{\sqrt{-1} \varphi}\right)_{\infty}}{\left(x_{k}^{-1} x_{l} q^{1+\alpha_{k}}|q|^{m} \rho_{0} e^{\sqrt{-1} \varphi}\right)_{\infty}}
$$

Hence we get the following estimates.

$$
\begin{aligned}
\left|\frac{1}{2 \pi \sqrt{-1}} \int_{\mathscr{C}_{m}} F(t) d t\right| & \leq\left|\frac{\rho_{m}}{2 \pi} \int_{0}^{2 \pi} F\left(\rho_{m} e^{\sqrt{-1} \varphi}\right) d \varphi\right| \leq \rho_{m} \underset{0 \leq \varphi \leq 2 \pi}{\operatorname{Max}}\left|F\left(\rho_{m} e^{\sqrt{-1} \varphi}\right)\right| \\
& \leq \rho_{m} M\left|q^{-1-\alpha_{0}-\alpha_{k}-\cdots-\alpha_{n}}\right|^{m} \leq \rho_{0} M\left|q^{-\alpha_{0}-\alpha_{k}-\cdots-\alpha_{n}}\right|^{m} .
\end{aligned}
$$

Thus we have

$$
\frac{1}{2 \pi \sqrt{-1}} \int_{\mathscr{C}_{m}} F(t) d t \longrightarrow 0 \quad(m \rightarrow \infty)
$$

when $\left|q^{-\alpha_{0}-\alpha_{k}-\cdots-\alpha_{n}}\right|$ is less than one. By the same argument we can show

$$
\frac{1}{2 \pi \sqrt{-1}} \int_{\tilde{\tilde{z}}_{m}} F(t) d t \longrightarrow 0 \quad(m \rightarrow \infty),
$$

when $\left|q^{\alpha_{0}}\right|$ is less than one. This completes the proof.

As a corollary of Lemma 2, a connection formula of the basic hypergeometric series, which tends to that of hypergeometric series as $q \rightarrow 1$, can be deduced. To state the corollary, we recall the definitions of the basic hypergeometric series ${ }_{2} \varphi_{1}$ and a $q$-analogue of the gamma function $\Gamma_{q}$ :

$$
\begin{aligned}
& { }_{2} \varphi_{1}(\alpha, \beta, \gamma ; x)=\sum_{n \geq 0} \frac{\left(q^{\alpha}\right)_{n}\left(q^{\beta}\right)_{n}}{\left(q^{\gamma}\right)_{n}(q)_{n}} x^{n}, \\
& \Gamma_{q}(x)=\frac{(q)_{\infty}}{\left(q^{x}\right)_{\infty}}(1-q)^{1-x} .
\end{aligned}
$$

Refer to [2], [5], [6], [11] for details.

\section{Corollary.}

$$
\begin{aligned}
{ }_{2} \varphi_{1}(\alpha, \beta, \gamma ; x) & =\frac{\Gamma_{q}(\gamma) \Gamma_{q}(\beta-\alpha)}{\Gamma_{q}(\beta) \Gamma_{q}(\gamma-\alpha)} \frac{\Theta\left(q^{a} x\right)}{\Theta(x)}{ }_{2} \varphi_{1}\left(\alpha, \alpha-\gamma+1, \alpha-\beta+1 ; q^{\gamma+1-\alpha-\beta} x^{-1}\right) \\
& +\frac{\Gamma_{q}(\gamma) \Gamma_{q}(\alpha-\beta)}{\Gamma_{q}(\alpha) \Gamma_{q}(\gamma-\beta)} \frac{\Theta\left(q^{\beta} x\right)}{\Theta(x)}{ }_{2} \varphi_{1}\left(\beta, \beta-\gamma+1, \beta-\alpha+1 ; q^{\gamma+1-\alpha-\beta} x^{-1}\right) .
\end{aligned}
$$

Proof. Consider the case of $k=1$ and $n=2$ in (6). Putting $\alpha_{0}=$ $1+\beta-\gamma, \alpha_{1}=\gamma-1-\alpha, \alpha_{2}=-\beta$ and $x_{1} \cdot x_{2}^{-1}=q^{\gamma-\alpha} x^{-1}$, one has

$$
\int_{0}^{1} t^{\alpha_{0}-1} \frac{(q t)_{\infty}\left(q^{\beta} x t\right)_{\infty}}{\left(q^{\gamma-\alpha} t\right)_{\infty}(q x t)_{\infty}} d_{q} t
$$




$$
\begin{aligned}
= & \frac{\Theta\left(q^{\beta+1-\gamma}\right) \Theta\left(q^{\beta} x\right)}{\Theta\left(q^{\alpha-\beta}\right) \Theta(x)} \int_{0}^{1} t^{\beta-\gamma} \frac{(q t)_{\infty}\left(q^{\gamma-\alpha+1} x^{-1} t\right)_{\infty}}{\left(q^{\gamma-\alpha} t\right)_{\infty}\left(q^{\gamma+1-\alpha-\beta} x^{-1} t\right)_{\infty}} d_{q} t \\
& +\frac{\Theta\left(q^{1-\beta}\right) \Theta\left(q^{\alpha} x\right)}{\Theta\left(q^{1+\alpha-\beta}\right) \Theta(x)} \int_{0}^{1} t^{\alpha-1} \frac{(q t)_{\infty}\left(q^{2-\beta} x^{-1} t\right)_{\infty}}{\left(q^{1-\beta} t\right)_{\infty}\left(q^{\gamma+1-\alpha-\beta} x^{-1} t\right)_{\infty}} d_{q} t .
\end{aligned}
$$

Thanks to the Jackson integral representation of the basic hypergeometric series

$$
{ }_{2} \varphi_{1}(\alpha, \beta, \gamma ; x)=\frac{\Gamma_{q}(\gamma)}{\Gamma_{q}(\alpha) \Gamma_{q}(\gamma-\alpha)} \int_{0}^{1} t^{\alpha-1} \frac{(q t)_{\infty}\left(q^{\beta} x t\right)_{\infty}}{\left(q^{\gamma-\alpha} t\right)_{\infty}(x t)_{\infty}} d_{q} t
$$

we obtain the required relation.

\section{§3. Solution to the connection problem}

Let $s=\max \left\{\left|x_{2}\right| x_{1}|,| x_{3}\left|x_{2}\right|, \cdots,\left|x_{n}\right| x_{n-1} \mid\right\}$. For $k=1,2, \cdots, n$, we have the following estimates, which are easily shown by the $q$-binomial theorem (4).

1) For $l=k, \cdots, n$,

$$
\int_{w_{k}} \Phi(t) d_{q} t=\int_{w_{l}} t^{\alpha_{0}-1} \prod_{j=k}^{n} \frac{\left(t / x_{j}\right)_{\infty}}{\left(q^{\alpha} t / x_{j}\right)_{\infty}} d_{q} t(1+O(s)) \quad(s \rightarrow 0) .
$$

2)

$$
\begin{aligned}
& \frac{\Theta\left(q^{1+\alpha_{0}+\alpha_{k}+\cdots+\alpha_{n}}\right)}{\Theta\left(q^{\alpha_{0}+\alpha_{k+1}+\cdots+\alpha_{n}}\right)} \int_{[0,1]} t^{-1-\alpha_{0}-\alpha_{k}-\cdots-\alpha_{n}} \prod_{j=k}^{n} \frac{\left(x_{j} x_{k}^{-1} q^{\alpha_{k}-\alpha_{j}} t\right)_{\infty}}{\left(x_{j} x_{k}^{-1} q^{\alpha_{k}} t\right)_{\infty}} d_{q} t \\
& \quad=\frac{\Theta\left(q^{1+\alpha_{0}+\alpha_{k}+\cdots+\alpha_{n}}\right)}{\Theta\left(q^{\alpha_{0}+\alpha_{k}+1+\cdots+\alpha_{n}}\right)} \int_{[0,1]} t^{-1-\alpha_{0}-\alpha_{k}-\cdots-\alpha_{n}} \frac{(t)_{\infty}}{\left(q^{\alpha_{k}} t\right)_{\infty}} d_{q} t(1+O(s)) \\
& \quad=\frac{\Gamma_{q}\left(1+\alpha_{k}\right) \Gamma_{q}\left(\alpha_{0}+\alpha_{k+1}+\cdots+\alpha_{n}\right)}{\Gamma_{q}\left(1+\alpha_{0}+\alpha_{k}+\cdots+\alpha_{n}\right)} q^{-\alpha_{0}-\alpha_{k}-\cdots-a_{n}}(1+O(s) \quad(s \rightarrow 0) .
\end{aligned}
$$

By the above estimates 1), the left hand side of (6) is

$$
\left(\int_{w_{k}} \Phi d_{q} t+\sum_{l=k+1}^{n} C_{k, l} \int_{w_{l}} \Phi d_{q} t\right)(1+O(s)) \quad(s \rightarrow 0),
$$

and by 2) the right hand side of (6) is

$$
\begin{aligned}
& \frac{\Gamma_{q}\left(1+\alpha_{k}\right) \Gamma_{q}\left(\alpha_{0}+\alpha_{k+1}+\cdots+\alpha_{n}\right)}{\Gamma_{q}\left(1+\alpha_{0}+\alpha_{k}+\cdots+\alpha_{n}\right)} q^{-\alpha_{0}-\alpha_{k}-\cdots-\alpha_{n}} \\
& \quad \times x_{k}^{\alpha_{0}} \prod_{j=k+1}^{n} \frac{\Theta\left(x_{j} / x_{k}\right)}{\Theta\left(x_{j} / x_{k} q^{\alpha_{j}}\right)}(1+O(s)) \quad(s \rightarrow 0) .
\end{aligned}
$$

Therefore, by Lemma 2, we obtain the following. 
LEMMA 3. Let $k=1, \cdots, n-1$ and $\tilde{W}_{k}$ be the q-interval $w_{k}+$ $\sum_{l=k+1}^{n} C_{k, l} w_{l}$. Then we have

$$
\begin{aligned}
\int_{\tilde{W}_{k}} \Phi(t) d_{q} t= & \tilde{C}_{k} \frac{\Gamma_{q}\left(1+\alpha_{k}\right) \Gamma_{q}\left(\alpha_{0}+\alpha_{k+1}+\cdots+\alpha_{n}\right)}{\Gamma_{q}\left(1+\alpha_{0}+\alpha_{k}+\cdots+\alpha_{n}\right)} q^{-\alpha_{0}-\alpha_{k}-\cdots-\alpha_{n}} \\
& \times x_{k}^{\alpha_{0}} \prod_{j=k+1}^{n}\left(\frac{x_{k}}{x_{j}}\right)^{\alpha_{j}}(1+O(s)) \quad(s \rightarrow 0)
\end{aligned}
$$

where

$$
\tilde{C}_{k}=\prod_{j=k+1}^{n}\left(\frac{x_{j}}{x_{k}}\right)^{\alpha_{j}} \frac{\Theta\left(x_{j} / x_{k}\right)}{\Theta\left(x_{j} / x_{k} q^{\alpha_{j}}\right)}
$$

Remark. The functions $\tilde{C}_{k}(1 \leq k \leq n-1)$ are pseudo-constant.

By using Lemma 3, we give the asymptotic behavior of fundamental solutions of the system (5) in the region $\left|x_{1}\right| \gg \cdots \gg\left|x_{n}\right| \gg 1$. In fact, if the variables $\alpha_{j}$ and $x_{j}$ are changed by $\alpha_{j}-1$ and $x_{j} q^{-1}$ respectively, then $\int_{w_{l}} \Phi(t) d_{q} t$ is transformed into $\int_{w_{l}} \Phi_{j}(t) d_{q} t$ for $1 \leq j, l \leq n$, and $\tilde{C}_{k}, C_{k, l}$ remain invariant. Hence if we put $\tilde{W}_{n}=w_{n}$, we have

$$
\begin{aligned}
& \left(\begin{array}{c}
\int_{\tilde{W}_{1}} \Phi_{1} d_{q} t, \cdots \cdots, \int_{\tilde{W}_{1}} \Phi_{n} d_{q} t \\
\cdots \cdots \cdots \cdots \cdots \cdots \\
\int_{\tilde{W}_{n}} \Phi_{1} d_{q} t, \cdots \cdots, \int_{\tilde{W}_{n}} \Phi_{n} d_{q} t
\end{array}\right)
\end{aligned}
$$

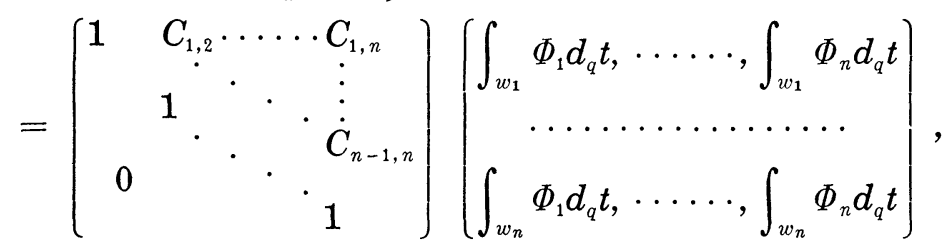

where

$$
\left\{\begin{array}{c}
\tilde{C}_{k} \frac{\Gamma_{q}\left(1+\alpha_{k}\right) \Gamma_{q}\left(\alpha_{0}+\alpha_{k+1}+\cdots+\alpha_{n}\right)}{\Gamma_{q}\left(1+\alpha_{0}+\alpha_{k}+\cdots+\alpha_{n}\right)} q^{1-\alpha_{0}-\alpha_{k}-\cdots-\alpha_{n}} \\
\quad \times x_{k}^{\alpha_{0}} \prod_{l=k+1}^{n}\left(\frac{x_{k}}{x_{l}}\right)^{\alpha_{l}} \quad(1 \leq k \leq n-1,1 \leq j \leq k-1), \\
\tilde{C}_{k} \frac{\Gamma_{q}\left(\alpha_{k}\right) \Gamma_{q}\left(\alpha_{0}+\alpha_{k+1}+\cdots+\alpha_{n}\right)}{\Gamma_{q}\left(\alpha_{0}+\alpha_{k}+\cdots+\alpha_{n}\right)} q^{1-\alpha_{0}-\alpha_{k}-\cdots-\alpha_{n}} \\
\quad \times\left(q^{-1} x_{k}\right)^{\alpha_{0}} \prod_{l=k+1}^{n}\left(\frac{x_{k}}{q x_{l}}\right)^{\alpha_{l}}(1 \leq k \leq n-1, j=k),
\end{array}\right.
$$




$$
\int_{\tilde{w}_{k}} \Phi_{j} d_{q} t \sim \begin{cases}\tilde{C}_{k} \frac{\Gamma_{q}\left(1+\alpha_{k}\right) \Gamma_{q}\left(\alpha_{0}+\alpha_{k+1}+\cdots+\alpha_{n}-1\right)}{\Gamma_{q}\left(\alpha_{0}+\alpha_{k}+\cdots+\alpha_{n}\right)} q^{\left(-\alpha_{0}-\alpha_{k}-\cdots-\alpha_{n}\right)+\alpha_{j}} \\ \quad \times x_{j} x_{k}^{\alpha_{0}-1} \prod_{l=k+1}^{n}\left(\frac{x_{k}}{x_{l}}\right)^{\alpha_{l}} \quad(1 \leq k \leq n-1, k+1 \leq j \leq n), \\ \frac{\Gamma_{q}\left(\alpha_{0}\right) \Gamma_{q}\left(\alpha_{n}+1\right)}{\Gamma_{q}\left(\alpha_{0}+\alpha_{n}+1\right)}\left(q x_{n}\right)^{\alpha_{0}} & (k=n, 1 \leq j \leq n-1), \\ \frac{\Gamma_{q}\left(\alpha_{0}\right) \Gamma_{q}\left(\alpha_{n}\right)}{\Gamma_{q}\left(\alpha_{0}+\alpha_{n}\right)} x_{n}^{\alpha_{0}} & (k=n, j=n) .\end{cases}
$$

The matrix on the left hand side of (7), which will be denoted by $\Xi_{e}$, may be taken as a fundamental solution of system (5) in the region $\left|x_{1}\right| \gg \cdots \gg\left|x_{n}\right| \gg 1$. Then the matrix expressed by

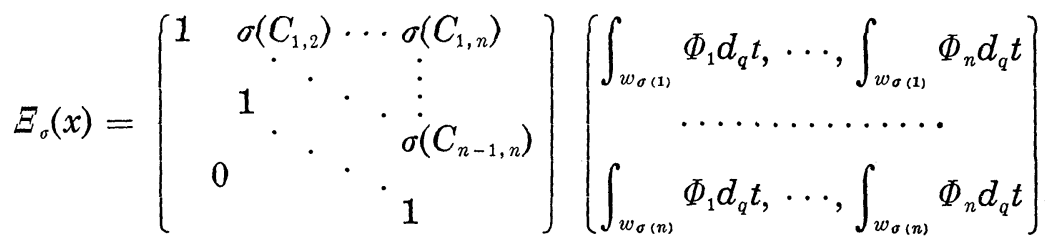

defines a fundamental solution in the region $\left|x_{\sigma(1)}\right| \gg \cdots \gg\left|x_{\sigma(n)}\right| \gg 1$, where $\sigma$ is an element of the symmetric group of order $n$. By the expressions (7) and (8), we can get the connection matrix $P_{\sigma, e}(x)$ between the solutions $\Xi_{e}(x)$ and $\Xi_{\sigma}(x)$. Indeed,

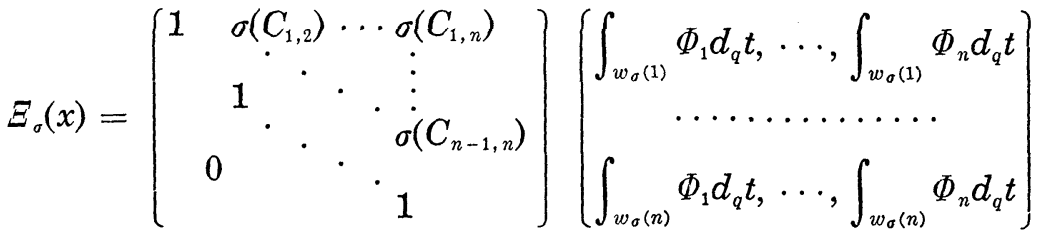

$$
\begin{aligned}
& =\left(\begin{array}{cccc}
1 & \sigma\left(C_{1,2}\right) & \cdots & \sigma\left(C_{1, n}\right) \\
& 1 & \cdot & \vdots \\
0 & \cdot & \cdot & \sigma\left(C_{n-1, n}\right) \\
& & & 1
\end{array}\right) \cdot S_{\sigma} \cdot \Xi(x) \\
& =\left(\begin{array}{cccc}
1 & \sigma\left(C_{1,2}\right) & \cdots & \sigma\left(C_{1, n}\right) \\
& 1 & \cdot & \vdots \\
& 1 & \cdot & \sigma\left(C_{n-1, n}\right) \\
0 & & \cdot & 1
\end{array} S_{\sigma} \cdot\left(\begin{array}{cccc}
1 & C_{1,2} & \cdots & C_{1, n} \\
& 1 & \ddots & \vdots \\
& 1 & \ddots & \dot{C}_{n-1, n} \\
0 & & \ddots & 1
\end{array}\right)^{-1} \Xi_{e}(x),\right.
\end{aligned}
$$

where $S_{\sigma}$ is a permutation defined by 


$$
S_{\sigma}\left(\begin{array}{c}
x_{1} \\
\vdots \\
x_{n}
\end{array}\right)=\left(\begin{array}{c}
x_{\sigma(1)} \\
\vdots \\
x_{\sigma(n)}
\end{array}\right),
$$

and the action of $\sigma$ on $C_{i, j}$ is defined by

$$
\sigma\left(C_{i, j}\right)=C_{i, j}\left(\alpha_{\sigma(1)}, \cdots, \alpha_{\sigma(n)} ; x_{\sigma(1)}, \cdots, x_{\sigma(n)}\right) .
$$

Hence we finally come to the main theorem of this paper.

THEOREM. The connection matrix between the solution $\Xi_{e}$ and $\Xi_{\sigma}$ is given by

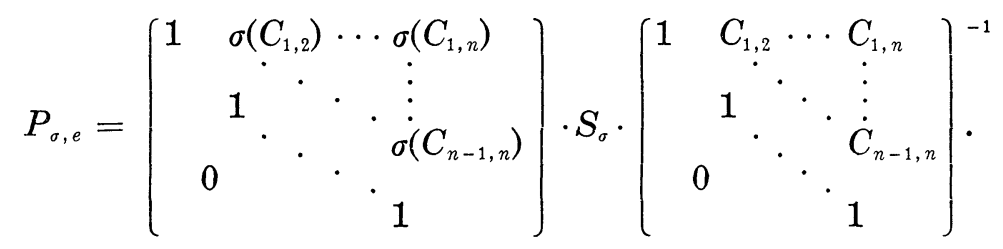

Remark. The connection matrices $P_{\sigma, e}$ satisfy the following cocycle conditions: $P_{\sigma, \tau}=P_{\sigma, e} P_{\tau, e}^{-1}, P_{\sigma \nu, e}=\sigma\left(P_{\tau, e}\right) P_{\sigma, e}$ for arbitrary two permutations $\sigma$ and $\tau$.

\section{§4. Appendix}

During the preparation of this paper $\mathrm{K}$. Aomoto suggested to the author the following question: to evaluate the integral (3) over an arbitrary $q$ interval $\mathscr{C}=[0, \infty(s)]$ of the second kind in terms of the integrals (3) over $w_{i}(1 \leq i \leq n)$. The answer is the following.

Theorem. Under the condition $\left|q^{-\alpha_{1}-\cdots-\alpha_{n}}\right|<\left|q^{\alpha_{0}}\right|<1$, we have

$$
\int_{[0, \infty(s)]} \Phi(t) d_{q} t=\sum_{l=1}^{n} R_{l} \int_{\left[0, x_{l}\right]} \Phi(t) d_{q} t
$$

where

$$
R_{l}=\left(\frac{s}{q x_{l}}\right)^{\alpha_{0}-1} \frac{\Theta\left(q^{\alpha_{l}+1}\right) \Theta\left(q^{\alpha_{0}+\cdots+\alpha_{n}-2} s x_{l}^{-1}\right)}{\Theta\left(q^{\alpha_{0}+\cdots+\alpha_{n}-1}\right) \Theta\left(q^{-1} s x_{l}^{-1}\right)} \prod_{j=1}^{n} \frac{\Theta\left(s / x_{j}\right)}{\Theta\left(q^{\alpha_{j}} s / x_{j}\right)} \prod_{\substack{j=1 \\ j \neq l}}^{n} \frac{\Theta\left(x_{j} x_{l}^{-1} q^{-\alpha_{j}}\right)}{\Theta\left(x_{j} x_{l}^{-1}\right)},
$$

and

$$
\Phi(t)=\prod_{j=1}^{n} \frac{\left(t / x_{j}\right)_{\infty}}{\left(q^{a_{j}} t / x_{j}\right)_{\infty}}
$$

Proof. Set 


$$
F(t)=\frac{\left(q^{\alpha_{0}+\alpha_{1}+\cdots+\alpha_{n}-1} / t\right)_{\infty}\left(q^{2-\alpha_{0}-\alpha_{1}-\alpha_{n}} t\right)_{\infty}}{(1 / t)_{\infty}(q t)_{\infty}} \prod_{j=1}^{n} \frac{\left(q x_{j} / q^{\alpha_{j}} s t\right)_{\infty}}{\left(q x_{j} / s t\right)_{\infty}}
$$

and

$$
\tilde{F}(t)=\frac{s^{\alpha_{0}}(1-q)(q)_{\infty}^{2}}{\left(q^{\alpha_{0}+\alpha_{1}+\cdots+\alpha_{n}-1}\right)_{\infty}\left(q^{-\alpha_{0}-\alpha_{1}-\cdots-\alpha_{n}+2}\right)_{\infty}} \prod_{j=1}^{n} \frac{\Theta\left(s / x_{j}\right)}{\Theta\left(s q^{\alpha_{j}} / x_{j}\right)} F(t) .
$$

Then the residues of $\tilde{F}(t)$ are expressed by the following Jackson integrals.

$$
\begin{aligned}
\sum_{-\infty<i<+\infty} \operatorname{Res}_{t=q^{i}} \tilde{F}(t) & =\int_{[0, \infty(s)]} \Phi(t) d_{q} t, \\
\sum_{i=0}^{\infty} \operatorname{Res}_{t=q x_{l} / s q^{i}} \tilde{F}(t) & =-R_{l} \int_{\left[0, x_{l}\right]} \Phi(t) d_{q} t,
\end{aligned}
$$

where $\operatorname{Res}_{t=x} F(t)$ denotes the residue of a function $F(t)$ at $t=x$. Therefore it remains to prove

$$
\sum_{-\infty<i<+\infty} \operatorname{Res}_{t=q^{i}} F(t)+\sum_{i=1}^{n} \sum_{i=0}^{+\infty} \operatorname{Res}_{t=q x_{l} / s q^{i}} F(t)=0 .
$$

We can show it in a similar way to that of Lemma 2; i.e. estimates of the integration of the function $F(t)$ on suitable cycles.

\section{Acknowledgement}

The author would like to express his gratitude to Professor Kazuhiko Aomoto for valuable súggestions.

\section{REFERENCES}

[1] C. R. Adams, On the linear ordinary q-difference equation, Ann. of Math., 30 (1929), 195-205.

[2] G. E. Andrews, " $q$-Series: Their development and application in analysis, number theory, combinatorics, physics, and computer algebra", CBMS Regional Conference Series in Math. 66 AMS, 1986.

[ 3 ] K. Aomoto, A note on holonomic $q$-difference system, Algebraic Analysis I, ed. by M. Kashiwara and T. Kawai, Acad. Press, 1988, 25-28.

[4] - private communications with the author.

[ 5 ] R. Askey, The q-gamma and $q$-beta functions, Applicable Anal., 8 (1980), 346-359.

[6] - Ramanujan's extensions of the gamma and beta functions, Amer. Math. Monthly, 87 (1980), 346-359.

[ 7 ] W. N. Bailey, "Generalized Hypergeometric Series", Cambridge University Press, 1935.

[8] G. D. Birkhoff, The generalized Riemann problem for linear differential and the allied problems for linear difference and $q$-difference equations, Proc. Amer. Acad. Arts and Sci., 49 (1914), 521-568. 
[9] R. D. Carmichael, The general theory of linear $q$-difference equations, Amer. J. Math., 34 (1912), 147-168.

[10] E. Heine, Uber die Reihe...., J. Reine Angew. Math., 32 (1846), 210-212.

[11] —_, Untersuchungen über die Reihe..., J. Reine Angew. Math., 34 (1847), 285328.

[12] F. H. Jackson, On $q$-definite integrals, Quart. J. Pure Appl. Math., 11 (1910), 193-203.

[13] K. Mimachi, A proof of Ramanujan's identity by use of loop integrals, SIAM J. Math. Anal., 19 (1988), 1490-1493.

[14] L. Pochhammer, Ueber eine lineare Differentialgleichung $n$-ter Ordnung mit einem endlichen singulären Punkte, J. Reine Angew. Math., 108 (1891), 50-87.

[15] L. J. Slater, "Generalized Hypergeometric Functions", Cambridge University Press, 1966.

[16] W. J. Trjitzinsky, Analytic theory of linear $q$-difference equations, Acta Math., 61 (1933), 1-38.

[17] E. T. Whittaker and G. N. Watson, "A Course of Modern Analysis", The Macmillan Company, 1943.

Department of Mathematics

School of Science

Nagoya University

Chikusa-ku, Nagoya 464-01, Japan 